\title{
2 The methodology of evidence-based research.
}

\subsection{RCTs and their importance for the methodology of EBM}

EBM is heavily based on quantifiable research and its results with the goal to achieve qualitative health care on the population level and on the level of the individual patient. The step from the population level to the individual in health care is a fairly complicated one and will be analysed in the following chapter, because it is also the most important one to free EBM from the criticism that it is not person-centred enough. In order to do so, it is necessary to look at how quantitative results are achieved and how the actual methodology of generating 'good' and 'usable' evidence functions. The methodology of evidence-based research is based on a hierarchical view of the quality of evidence. The actual evidence-hierarchy will be explained in detail in the chapter about evidence and epistemology. This chapter will focus on randomised controlled trials (RCTs) as those medical firstin-man trials which play a major role in the generating of evidence. RCT's are medical experiments that are designed to test safety, risk, efficacy and effectiveness, normally in this order, of novel treatments like drugs, surgery, and even acupuncture and physiotherapy.

The results of RCTs inform most medical and health policy decisions, since they are often considered to be the best option to arrive at robust and usable evidence. The following chapter will closely look at RCTs and will assess if they really are as fail-safe in producing 'good' evidence as they are made out to be.

On the very top of the overall hierarchy of evidence, including steps to assess evidence, are systematic reviews, or meta-analyses, because they pool and assess the available data, generated from RCT's and often from well-conducted cohort and observational studies, and make it comparable. The best-known institution for systematic reviews and meta-analyses is the Cochrane Collaboration and the Cochrane Library. ${ }^{35}$ Archie Cochrane could be called one of the founding fathers of EBM and epidemiology. He argued that it was vital, especially with sparse resources, that only those treatments are used which have shown beyond a doubt to be effective. He greatly favoured RCTs as a means to arrive at this goal. ${ }^{36}$ Cochrane reviews are instrumental in making evidence-based practice manageable

35 Cochrane Collaboration. http://www.cochrane.org. Last accessed on January $23^{\text {rd }}, 2020$.

36 Cochrane, trusted evidence, informed decisions, better health. http://www.cochrane.org/aboutus/our-name. Last accessed on January $23^{\text {rd }}, 2020$. 
for the evidence user. The literature about medical evidence is so vast that it is vital to have succinct reviews which pool the most robust evidence and are able to give short and precise statements about the pros and cons of the treatments in question. Meta-analyses and systematic reviews are not without faults however, since they can only be as good as the initial data provided. The Cochrane Collaboration, and like-minded organisations, strive diligently to regularly assess new data about topics of already conducted reviews in order to keep the evidence as up to date, and as manageable, as possible. Lesser known organisations are the Joanna Briggs Institute based in Australia, the "Centre for review and dissemination" and the EPPI Centre. ${ }^{37}$ Individual scientists and authors can also conduct systematic reviews and have them published. Yet again, even the amount of meta-analyses and systematic reviews about each single diagnosis is staggering and not manageable in its entirety for any physician or clinician. However, because of their special training, most clinicians can assess the relevance of the research pretty quickly and focus on the most important parts for their individual patients. Increasingly, these skills are already taught in medical schools. ${ }^{38}$

In this chapter the focus lies on the methodological and ethical problems of RCT's, since they are still viewed as the most important tool in the arsenal of conducting meaningful medical research and are therefore the cornerstones of EBM. For the longest time it was argued that the results from RCTs were the only admissible evidence in EBM. ${ }^{39}$ In the last decade however, with growing criticism towards EBM and its methods, it has been acknowledged that other forms of medical evidence can play an equally valuable role, especially when and where the individual patient is concerned. ${ }^{40}{ }^{41}$ But not all evidence is automatically 'good' evidence and even if it is considered to be 'good' the question remains: for whom?

\subsubsection{Scientific goals of RCTs}

The overall scientific goal of RCTs is to establish the absolute risk reduction, ARR, or inversely, the number-needed-to-treat, NNT, of patients with the novel treatment compared to standard or placebo. These numbers stand for the efficacy of the novel treatment.

37 Library Guides: Subjects, Services and Resources. http://libguides.rgu.ac.uk/c.php?g=536793\& $\mathrm{p}=4389919$. Last accessed on January $23^{\text {rd }}, 2020$.

38 Jorgen Nordenstrom. (2007). Evidence-Based Medicine in Sherlock Holmes' Footsteps. Oxford: Blackwell Publishing.

39 Gordon Guyatt, et.al. (1992): 2420.

40 Jo Rycroft-Malone, Kate Seers, Angie Titchen et.al. (2004). "What counts as evidence in evidence-based practice?" in Journal of Advanced Nursing. 47(1): 81-90.

41 Trisha Greenhalgh, et.al. for the evidence-based renaissance movement. (2014). 
I am using the example for NNT and ARR of the Centre for Evidence-Based Medicine Oxford here due to its conciseness: "The absolute risk reduction or absolute effect is the amount by which your therapy reduces the risk of the bad outcome. For example, if your drug reduces the risk of a bad outcome from 50 per cent to 30 per cent, the ARR is:

$\mathrm{ARR}=\mathrm{CER}-\mathrm{EER}=0.5-0.3=0.2(20$ per cent $) .{ }^{.42}$

CER stands for Control Event Rate and EER for Experimental Event Rate.

The NNT is the inverse to the ARR and is always rounded up to the nearest number since whole patients are treated, not fractures.

$\mathrm{NNT}=1 / \mathrm{ARR}$

For the example above that means that:

$\mathrm{NNT}=1 / \mathrm{ARR}=1 / 0.2=5$

The NNT stands for the number of patients that need to be treated in order to improve one patient. Therefore the ideal NNT is 1, as in one patient. The higher the NNT is, the less patients benefit from the novel treatment. However, even a treatment with a high NNT can be useful in particular circumstances, especially when no other treatment for this particular patient is available. It is very context dependent if a treatment with a high NNT can still be deemed acceptable. The experience of the clinician can guide the judgement call if a treatment with a high NNT might still be useful in a particular case. In trials about disease prevention, the NNT is often allowed to be higher than in trials testing singular treatments or drugs. ${ }^{43}$

Additionally to the efficacy of the novel treatments, RCTs are also, and even more importantly, conducted to assess the safety of a novel treatment. This is the special focus of early phase RCTs. If the safety cannot be established, then no further RCTs will be performed. Efficacy does not trump safety!'Safety' however should not be confused with 'overall risk' of a treatment. The risk/benefit factor needs to be established in a trial. If the actual benefit of a treatment outweighs its risk/s, then further RCTs should be conducted to assess how far they actually do so and if it is still reasonable to use a treatment even though it contains a certain amount of risk.

42 CEBM: Centre for Evidence-Based Medicine, Oxford. "Definition of ARR and NNT." http:// www.cebm.net/2014/03/number-needed-to-treat-nnt/. Last accessed on January 23rd, 2020.

43 Katja Suter, Matthias Briel and Judith Günther. (2015). "Number needed to treat (NNT) and Number needed to harm (NNH): weitere Abkömmlinge der Vier-Felder-Tafel." in Medizinische Monatszeitschrift für Pharmazeuten 38(3): 103-106. 
RCTs come in multiple forms and with various research protocols attached to them, always depending on what it is that is to be tested. Trials testing non-drug treatments are slightly different to those testing drugs, and those testing preventing measures like vaccines are yet again slightly different. However, there are some aspects that they all have in common and these will be the focus of the discussion. RCTs are so-called 'first-in-man trials and are conducted in different phases and with a different amount of participants necessary, exactly to assess overall safety first and subsequently, efficacy, risk and effectiveness.

RCT's are set up in a way in order to arrive at "ideal" results, i.e. results that are reliable and robust. Since it is good scientific practice never to rely on the results of single trials, most treatments are tested in multiple clinics, in multiple RCTs, sometimes with differing, sometimes with the same research protocols. The results of one RCT need to be reproducible, and preferably more than once. ${ }^{44} \mathrm{Re}$ producibility of results leads to scientific acceptance, and to the results to be possibly externally valid and therefore of relevance to the actual target population. However, a multitude of trials and trial data, often running over hundreds of pages, is not usable as such for the actual evidence user. ${ }^{45}$ That is why systematic reviews and meta-analyses are so important, because they assess the available data, dismiss badly conducted trials and make the results easier to 'digest.' Therefore, they are a vital part of EBM, because they make evidence-based practice possible in the first place.

If RCTs are set up correctly, they are considered to be internally valid, which means that they yield correct and rigorous results about the treatment for the actual trial population under test and have used the correct methods to do so. They are therefore comparable to an experiment in a laboratory setting, insofar as that they adhere to the same form of rigorousness. However, that internal validity does not yet make RCTs also viable for the actual target population needing that specific treatment for a that specific ailment, let alone for the individual patient. On the contrary, a very high internal validity can lead to a quite low external validity. ${ }^{46}$ If and how this specific problem can be solved will be discussed later.

The quality of the results of RCT's is based on the validity of the trials and the validity of the RCT's is based, more often than not, on successful randomisation and on successful blinding. Randomisation and blinding are the two main methodological features of RCTs that set them apart from trials like cohort studies and observational studies, both of which will be evaluated as part of the hierarchy

44 J. Shao and SC. Chow. (2002). "Reproducibility probability in clinical trials." in Statistics in Medicine. 21(12):1727-42.

45 Jorgen Nordenstrom. (2007):

46 Nancy Cartwright. (2007). “Are RCTs the Gold Standard?” in BioSocieties. 2 (1) (Special Issue: The Construction and Governance of Randomised Controlled Trials). 11-20. 
discussion, and they are the two features which need to be maintained throughout the trial to really render robust results.

\subsubsection{Publication of all trial data}

In order to successfully assess RCTs and to conduct meta-analyses, the results of all RCTs need to be published in a coherent and complete form, which unfortunately they are not, at least in many cases ${ }^{47}$ To establish reporting standards, the CONSORT Group, a consolidated group of two research groups, both aiming at a standardisation of trial reporting, merged in 1996 and published the first CONSORT Statement in 2007 and a revised form in $2010 .{ }^{48}$ The CONSORT statement and tool consists of a flow chart and checklist for authors of RCTs to control if all necessary information is written up and all data is included. Many academic journals prefer that the trial write-up is done according to CONSORT or even stipulate the necessity for authors to adhere to CONSORT before publishing any research results. CONSORT however does not judge the methodology of RCTs. It only aims at good reporting standards to prevent reporting bias. The value of the methodology of RCTs however lies in their set-up and in the quality of said set-up which starts with randomisation.

\subsubsection{Introduction of the basic methods of RCTs}

As the name already indicates, the most prominent and important feature of RCTs is 'randomisation.' Randomisation means that trial participants are divided into two different, but equal, groups. There are different methods of randomisation available and these will be discussed throughout the chapter. In its most basic, but also most usual form one group receives the treatment under trial and the other group receives either the standard treatment or a placebo. Placebos can be either inactive or active and both pose their own problems, ethical and methodological, as do placebo-controlled trials as a whole. These 'special' trials will be discussed at length later on.

The main question here is if randomisation really has the methodological virtue to make the groups equal and what role confounding factors play for answering that question. Blinding is the second most important step in the set-up of RCTs and means that at least two or more groups involved in the trial do not know in

$47 \quad$ Ben Goldacre. (2012): 81.

48 The Consort Statement. http://www.consort-statement.org/ Last accessed on January $23^{\text {rd }}, 2020$. 
which groups the participants are randomised into ${ }^{49}$ Blinding is supposed to control for confounders such as selection - and allocation bias, i.e. the researcher consciously or unconsciously putting participants into either the control or the treatment arm based on where they will do better. The other case which is to be avoided is that the participant knows which treatment he will receive in order to minimise expectations toward the treatment. The difficult part in any blinded trial is to maintain the blinding. At least one party can be blinded but the goal is to blind all parties involved in a trial. In trials were treatments such as pills or injections are under test, blinding of a large group of people involved is possible, even to the extent that the outcome assessors, those who receive the raw data and evaluate it, are blinded. In trials concerning surgical procedures it is much more complicated to maintain the blinding, since the surgeon at least needs to know if he is supposed to perform a real or a sham surgery. The same is true for all trials testing treatments where at least the dispensing physician or health practitioner needs to know which treatment he or she is administering. Acupuncture would be such an example where it is impossible to blind the acupuncturist. ${ }^{50}$

\subsection{The different phases of RCTs}

Before a drug or treatment is tested on human beings, it is developed and rigorously tested in the laboratory. Often, in the later stages of the development, these tests and trials involve laboratory animals, such as rats or monkeys for example. The necessary requirement is most often that the DNA has a special similarity to that of humans, or that some other feature is close enough to humans to make the results of animal studies usable in later human studies. Animal studies however suffer the same problems that RCTs in humans do. They can be flawed through observation or publication bias and through the lack of external validity. It is acknowledged that they are and were necessary to further the understanding of the mechanisms of disease but are less well equipped to reliably inform about the effectiveness and safety of treatments. ${ }^{51}$

In these animal trials, randomisation and blinding is not absolutely necessary and often hard to perform because the sample sizes can be too small. It is however important to mention that in theory, and even sometimes in practice, RCTs can be performed in animal studies where the groups are sufficiently big enough and can

49 Jeremy Howick. (2011): 63.

50 Edzard Ernst and Simon Singh. (2008). Trick or Treatment? The undeniable facts about alternative medicine. London: Transworld Books: 67.

51 H. Bart van der Worp, David Howells, et.al. (2010). "Can Animal Models of Disease Reliably Inform Human Studies?" in PLoS Med 7(3): 1. 
be meaningfully randomised and the observer could even be blinded as to the intervention, just recording the observations without attaching any results. ${ }^{52}$ Since 2010 the ARRIVE guidelines, as an equivalent to the CONSORT guidelines, have been adopted to make animal research and the publication of animal research more transparent and to make it liable to ethical considerations. ${ }^{53}$

However, when there is talk of RCTs in the medical setting, it is necessary to assert that it is almost always based on trials involving humans. So when we talk about RCTs here, it is about phase 0 to IV trials, those following the laboratory stage of the research, involving human beings. The most common RCTs are performed in phases I to III, phases 0 and IV are rarely performed. However, especially phase IV trials, those which are conducted after the market approval of the treatment, are of vital importance for the safety assessment of a drug, because they can be conducted over a lengthy period of time, in many patients of the actual target population, and side effects can be more easily detected. ${ }^{54}$

\subsubsection{Phases of RCTs according to Benedetti: ${ }^{55}$}

Phase 0 trial: These trials are fairly rare, mostly conducted in cancer research and are only involving a very small number of participants who are usually suffering from the disease. The aim of phase 0 trials is to establish safety and the potential of the drug to reach the target area depending on the dosage. Therefore it is essential that the participants are suffering from the disease in question. The novel treatment is rarely tested against a placebo.

Phase I trials: These are for most drugs and treatments the actual first-in-man trials. They are conducted, usually, on a very small number of healthy participants. The main questions are 'safety of dosage', possible side effects, and the bodies reaction to the drug. The novel treatment is rarely tested against placebo.

Phase II trials: These trials try to answer the same questions as in a phase I trial, but are conducted with a larger number of participants, often patients with the actual disease or illness in question. Since a base-safety of the drug is established after successful phase I trials, efficacy of the drug plays a bigger role than safety.

52 Beverly Muhlhausler and Frank Bloomfield, et.al. (2013). "Whole Animal Experiments Should Be More Like Human Randomized Controlled Trials." in PLoS Biology, 11(2).: 1.

53 Beverly Muhlhausler and Frank Bloomfield, et.al. (2013).

54 Fabrizio Benedetti. (2014). Placebo Effects. 2nd Ed. Oxford: University of Oxford Press, Kindle Version: Chapter 1.2.1 Placebos are the tenet of the randomised, double-blind, placebo-controlled trial design.

55 Fabrizio Benedetti. (2014): Chapter 1.2.1 Placebos are the tenet of the randomised, double-blind, placebo-controlled trial design. 
Phase III trials: These are the last trials before a possible licensing and market approval of the drug. They are preferably conducted with a huge number of participants and the novel treatment should be tested against the standard treatment, and only against placebo if no standard is available. Safety, efficacy and effectiveness of the novel treatment are again the main questions for the researchers. Side effects are observed over some length of time.

Phase IV trials: These trials are conducted after the licensing and market approval of the novel treatment. They are most often observational studies which gather information of the long-term risk and safety of the drug as it hits its actual target population and therefore play a part in establishing external validity. Unfortunately phase IV trials are fairly rare, even though they could be conducted, even as RCTs, quite easily, if GPs and clinicians were allowed to gather the appropriate data in their daily practice. ${ }^{56}$

The trials as they are described above are idealised versions of RCTs. The number of participants per trial, especially if it is a small number, contributes in a big way to the problems of RCTs. Phase II and III trials require, to be performed 'correctly,' a fairly large number of participants. The more participants the better, since the stratification of possible confounders, factors that influence the trial results, is better guaranteed and even rare side-effects are picked up more easily, if they are picked up at all in the given time. The obvious problem is the recruitment of this larger number of participants for many different reasons which will be discussed throughout the chapter. Sometimes the disease is simply so rare that there are just not as many patients available as a trial would actually need. In these cases provisions are done for smaller trials and the smaller number of participants is not interpreted as a flaw of the trial. ${ }^{57}$ In most cases however there are just not enough volunteers. Either because patients are not made aware that a trial is conducted for which they would be eligible or they are not 'ideal' enough, which means they are too ill or have too many co-morbidities. Or they simply do not want to participate in a trial, fearing that they receive the lesser treatment or lesser care ${ }^{58}$ However, in many trials the care is even better in the research setting than it would be in the regular setting, since the participants are under close supervision and are controlled much more regularly than a patient in a normal GP practice would be. That is especially true, and alarmingly so, in trials that are conducted in the developing

56 Ben Goldacre. (2012): 225-241.

57 Niklas Juth (2014). "For the Sake of Justice: Should We Prioritize Rare Diseases?" in Health Care Annals.

58 Yvonne Brandberg, Hemming Johansson, Mia Bergenmar. (2016). "Patients' knowledge and perceived understanding -Associations with consenting to participate in cancer clinical trials." in Contemporary Clinical Trials Communications 2: 6-11. 
world. Trials are less expensive to conduct there and participants are presumably easier to find, because the level of overall care is so bad that research settings provide the one possibility for many patients to even have some form of treatment. ${ }^{59}$

\subsection{Confounding factors and their influence on trial results}

Confounding factors are those factors, like age, gender, overall health, weight, pregnancy, existing illnesses, chronic diseases, etc., but also many forms of bias, that can severely change the outcome of a trail. The attempt to control for confounding factors is what makes randomisation and blinding so important for medical trials. Confounding factors are divided into known and unknown confounders and both types can differ significantly from trial to trial, depending on the treatment or drug under test. ${ }^{60}$ Therefore, there is no exhaustive list of possible confounders and every investigator has to conclude from previous research which confounders are relevant for the trial and subsequently need to be controlled for. This before-hand control however can only work for known confounders. Unknown confounders make control mechanisms, such as randomisation and blinding even more necessary.

Howick explains confounding factors by labelling them with three properties. First: "the factor potentially affects the outcome." Second. "the factor is unequally distributed between experimental and control group" and third: "the factor is unrelated to the experimental intervention." ${ }^{\prime 61}$ The most important feature of confounding factors is that "each confounding factor provides a potential alternative explanation for the results of a clinical trial." 62 This chapter will therefore aim to explain why the control for possible confounders is on the one hand important for the overall validity of the test results and therefore desirable and on the other hand not always possible and not always necessary, even if the above explanation of confounding factors is correct.

Sometimes, mostly in phase IV trials, does it become apparent that confounding factors were responsible for the overall outcome of previous trials. In some cases the deviation between the results of a drug or treatment before market approval and afterwards is so big that the drug will be, and needs to be, taken off the

59 Sonia Shah. (2008). Am Menschen Getestet! Wie die Pharmaindustrie die Ärmsten der Welt für Medikamententests missbraucht. München: Redline Wirtschaft.

60 Jeremy Howick. (2011): 34.

61 Jeremy Howick. (2011): 77. Howick modifies this third property with regard to side effects. In the moment I will let it stand as is and will come to the point about side effects later. "the confounding factor must be unrelated to the positive characteristic effects of the experimental treatment on the target disorder (as opposed to side effects)." Jeremy Howick. (2011): 36. 
market. A well-known example of such a case, albeit without a phase IV trial was running, is the thalidomide scandal. Thalidomide was a drug manufactured in the 1950s and 1960s in Germany and given to many expecting mothers to treat nausea and fatigue. ${ }^{63}$ After the drug was on the market for quite some time it was discovered that it caused malformation of the limbs, called phocomelia, in newborns. Thalidomide was subsequently taken off the market, but only after a huge scandal ensued in Europe, the United States and Canada ${ }^{64}$ It is now in use again for certain types of cancer and for rare complications in leprosy, but pregnant women are specifically warned not to use the drug. ${ }^{65}$ The Thalidomide scandal has influenced, and changed, how drugs and treatments are tested today and how the marketing approval of new drugs is governed to prevent similar 'mistakes' in the future.

Medical scandals show how necessary it is to test the safety of a new treatment or drug, long before it reaches the open market, and to stay diligent even after it is approved. And in order to securely arrive at a conclusion about safety, all the other factors that could influence the outcome must be eliminated in the best possible way. That makes it so important to control for all confounders, known and unknown, as good as possible. As will become obvious, randomisation and blinding are far from perfect to always and reliably control for unknown confounders and many scientists seem to agree about their imperfectness without presenting valid alternatives. Therefore it is important to study the shortcomings of these control mechanisms and to try and improve them, instead of vilifying RCT's in general, as some seem to do. ${ }^{66}$

So in order for RCT's to be internally valid and to arrive at "ideal" results, they have to control for confounders and use randomisation and blinding to do so. ${ }^{67}$ The two methods go hand in hand with each other but need to be examined somewhat separately because they each have their inherent problems and strength.

63 Imogen Evans, Hazel Thornton et.al. (2011): 4.

64 Jack Botting. (2002). "The History of Thalidomide." in Drug News \& Perspectives. 15(9): 604.

65 Teru Hideshima, Dharminder Chauhan et. al. (2000). "Thalidomide and its analogs overcome drug resistance of human multiple myeloma cells to conventional therapy." in Blood, Vol. 96:2943-2950.

66 John Worrall. (2010). "Do we need some large, simple randomized trials in medicine?" in M. Suarez, M. Dorato and M. Redei (eds). EPSA Philosophical Issues in the Sciences. Dordrecht: Springer.

67 Jeremy Howick and some other philosophers of science would prefer to call "blinding" masking, because blinding is a derogatory term towards visually impaired patients. Since most articles and books about RCT's still use the term "blinding", that is what I will use, without any derogatory intent. 


\subsubsection{Randomisation}

Randomisation will be explained first, since it is understood as playing the most important role in the control for possible confounders. ${ }^{68}$ Randomisation in its most simple form means that patients who are willing to participate in a trial are selected into two groups, the treatment group that receives the novel drug or treatment and the control group that receives the standard treatment or a placebo. The selection process is randomised, most often according to a numerical pre-specified code that is not known to the researcher or the participant. Therefore, neither know in which treatment arm the participant is randomised into. Eligible participants are most often randomised via an independent agency which has produced the randomisation codes. The researcher receives a number for the patient that is matched either for control or for treatment. The random numbers are generated via a computer program and coded before they are given out. Although it sounds very complicated, the method is very close to throwing a dice or flipping a coin. ${ }^{69}$ The main reason that computer generated numbers are used is that there is less of a possibility to manipulate the process. The method of computer based randomisation can be compared to a lottery. Most often in practice, after the computer-generated lottery has run, envelopes are prepared which "contain randomly generated instructions about which group to assign the next patient." ${ }^{.70}$ Here blinding and randomisation go hand in hand. The researcher receives the envelope but since he does not have sufficient information to decipher the code, he is blinded to the intervention that the participant is randomised into.

There are a number of softwares available on the internet which are fairly easy to use and can be used for multiple types of randomisation. One of those often used by medical statisticians is http://www.graphpad.com/quickcalcs/index.cfm. Graphpad even goes beyond that and offers different statistical methods to arrive at and calculate results.

In EBM both single and cluster randomisation can be used. ${ }^{71}$ In evidencebased policy (EBP), cluster randomisation is most often the method of choice, since policies are never implemented on an individual level. Cluster randomisation means that entire groups of people are randomised as groups, not divided into individuals. Single randomisation simply means randomisation at the individual patient level, with the methods described above. In EBM cluster randomisation could

68 John Worrall. (2004). "Why there's no cause to randomize." Technical Report in Causality: Metaphysics and Methods. CPNSS: 3.

69 K.P. Suresh. (2011). "An overview of randomization techniques: An unbiased assessment of outcome in clinical research." in Journal for Human Reproductive Sciences. 4(1): 9.

70 Jeremy Howick. (2011): 43.

71 BetterEvaluation: Sharing information to improve evaluation. http://betterevaluation.org. Last accessed on January $23^{\text {rd }}, 2020$. 
for example mean that the participating clinics would be randomised, and the patients would be treated with either the novel or the standard treatment, depending in which clinic they would be treated at. Another form of cluster randomisation is also called block randomisation and is sometimes used when it is important to have equal sample-sizes. Block-randomisation however is not very good in eliminating confounding factors. ${ }^{72}$ Cluster randomisation can achieve that, if enough patients in each clinic participate in the trial.

To eliminate known confounders before the trial, especially when the number of participants is high, stratified randomisation can be used. It starts with a type of block randomisation in which participants are assigned to different blocks depending on the confounder. "After all subjects have been identified and assigned into blocks, simple randomization is performed within each block to assign subjects to one of the groups." ${ }^{73}$ This method however only works when all participants are selected before the trial. If they are selected on a continuous basis while the trial is running, then regular single randomisation should be used. ${ }^{74}$ Cases in which stratification on the individual level is asked for are normally involving certain aspects of a specific disease. An example here can be any cancer that can appear with or without metastases. A novel treatment can yield positive results in both cases, but the participants should be randomised according to the presence or absence of metastases, so that the control and the treatment arm contain a fair number of both types of cancer patients. Therefore both 'types' of cancer are used as strata and the participants within the strata are then randomised into control and treatment arms. ${ }^{75}$ However, an approach like this is obviously only workable with very few strata, otherwise the subgroups would be too small. Machin claims that "For continuous prognostic variables such as age, stratification can only be carried out when these variables are divided into categories. [blocks] ... Although age (or some other continuous variable) may be prognostic for outcome, it is usually preferable not to stratify for this but to record the information for each patient and take account of this in a retrospective sense at the analysis stage." 76 However "controlling' for confounders during the analysis stage, when all the data is gathered, can be very complicated and can lead to false results. Therefore it would be better to use some pre-stratification and thorough randomisation in order to control for known confounders right from the beginning of the trial.

72 K.P. Suresh. (2011): 10.

73 K.P. Suresh. (2011): 10.

74 BetterEvaluation Pg. 2.

75 David Machin and Peter M. Fayers. (2010). Randomized Controlled Trials, Design, Practice and Reporting. Oxford: Wiley-Blackwell: 101.

76 David Machin and Peter M. Fayers. (2010): 102. 
In systematic reviews, all types of randomisation can be compared, but it should be specified which type of randomisation was used in order to better evaluate the outcome. An example where specification was necessary is a meta-analysis of trials in low- and high-income countries about antenatal care for pregnant women. The women in the high-income countries were randomised individually and the women in the low-income countries were randomised in clusters. The trials were set-up to evaluate how much care was necessary for women with a low-risk pregnancy and if the number of antenatal visits could be decreased. ${ }^{77}$ One main reason between the differences in randomisation in this example is the difference between high- and low-income countries. In high-income countries, 'contamination' is less likely. 'Contamination' can occur when participants communicate with each other and find out in which arm they belong and what they potentially miss out on. Contamination can lead to participants leaving trials and seeking for a higher level of care elsewhere. Even though pregnant women speak with each other and might compare their levels of care in high-income countries, the overall level of care is high and being in the test arm of a trial, especially in this case, does not pose a greater risk to either mother or child, as was shown in the sub-group analyses of high-income countries in which no more perinatal death occurred than in standard care. ${ }^{78}$ In low-income countries the risk of contamination was deemed higher. Cluster randomisation of the clinics was used to prevent this, since all patients in one clinic are either treated with the novel or the standard treatment. However, possible hidden 'individual' confounders are not controlled for in cluster randomisation. Therefore, it is impossible to directly compare trials with individual and with cluster randomisation in most cases, simply because possible confounders cannot be equally controlled for. One obvious confounder in the example is already the difference in income and overall care given. Perinatal death were higher in the test-arm in low income countries, because conditions which would have led to the admission into neonatal intensive care were not recognised at all or too late. One outcome of the trials and the subsequent meta-analysis was that all women were less satisfied with fewer prenatal visits, regardless of low- or highincome countries.

Even single randomisation can be problematic, if it is not done correctly, since the different methods of single randomisation, if they are not done as described above, can be easily manipulated. One very questionable method is the Zelen method of randomisation, because participants are only asked for their consent to participate in a trial after the randomisation has already happened. ${ }^{79}$ The

77 T. Dowswell and G. Carroli. (2010). "Alternative versus standard packages of antenatal care for low-risk pregnancy." in Cochrane Database of Systematic Reviews, Issue 10. Art.

78 T. Dowswell and G. Carroli. (2010).

79 Marvin Zelen. (1979). "A new design for randomized clinical trials." In New England Journal of Medicine 300: 1242-1245. 
method can lead to obvious selection bias and to early drop-out rates if and when the participants are questioning the trial and opt out to use the standard treatment. A choice denied to those patients in the control arm of the trial, because there were not informed at all about their trial participation. When Zelen wrote his randomisation proposal, such an approach would still have been possible. Today however, participants have to consent to the use of their data. Therefore they need to be informed about all the possible choices and the Zelen method is ethically and methodologically problematic. The latter is the case because neither the participant nor the researcher are blinded as to the intervention which can lead to selection and observation bias. The former because participants in the control arm are left in the dark as to the treatment possibilities.

"Randomised plays the winner" is another form that was and still is used to randomise participants on an ongoing basis. ${ }^{80}$ It functions in a way that if one treatment is more effective than the other, then more participants will receive the "effective" treatment and this can result in false-negative or false-positive overall results of the trial, because the patients are not divided equally anymore between the arms and confounders and other deviations in the participant population can severely change the results.

Howick points out the problem of "pseudo-randomisation" which occurs when a randomisation procedure is too easy to decipher. An example would be if every other patient, who would be eligible for a trial, would be put in the control arm. If this type of randomisation would be used, blinding of the different parties could be easily subverted. ${ }^{81}$ Howick makes the argument that knowledge about the allocation does not need to undermine the validity of the trial, if the randomisation is not tampered with. ${ }^{82}$ Further down the argument, he however admits that "allocation bias and self-selection bias can become worrisome again." ${ }^{83}$ Howick is convinced that proper blinding is simply not possible and should therefore not have as much methodological value as Worrall and others are allowing it. However, blinding seems to be the only method to successfully rule out self-selection and allocation bias. I will come back to this problem later in the chapter.

80 Elbourne, D. Field, D. Mugford, M. (2002). "Extracorporeal membrane oxygenation for severe respiratory failure in newborn infants (Review)". The Cochrane Collaboration. Wiley.

81 John Worrall. (2003). "What Evidence in Evidence-Based Medicine." in Causality: Metaphysics and Methods. CPNSS: 14.

82 Jeremy Howick. (2011): 44.

83 Jeremy Howick. (2011): 44. 


\subsubsection{Confounding factors during trials:}

If, despite all efforts to the contrary, confounders do appear during the trial which are influencing the results, the trial needs to be changed in some way.

An obvious solution would be re-randomisation or stratification, taking the new known confounder into account. Re-randomisation however is hardly ever discussed since the option of choice is to match the results post trial or to abandon the trial altogether. Since re-randomisation sounds like the most common-sense approach, I will briefly discuss the reasons why it is not a viable solution to the problem. In re-randomisation it could happen that the previously gathered data cannot be used anymore. The whole trial would have to be organised again, preferably with new participants and a new protocol. If the same participants would be re-randomised, a wash-out period would be needed, since almost all drugs stay in the body for a certain amount of time. Additionally, the treatment or drug would have already shown benefits, that for example in a placebo-controlled trial only appeared in the treatment arm. Blinding would be nearly impossible to maintain. To add new participants to the 'old' participants would be equally complicated, even if a wash-out period is maintained, because the reactions to the new treatment might be different and again blinding would not be maintained. The participant base is therefore too contaminated to successfully extrapolate useful research results.

Another problem of re-randomising is that it is time-consuming and therefore costly. RCTs however are extremely costly to begin with and the number of participants, regardless of how many there are, is usually too small anyway. Research hospitals and clinics have provided facilities and staff for a certain amount of time to run a trial and in the case of re-randomisation all that would be needed again, again at substantial costs to the research facility. Since in many RCTs it is unsure if the trial leads to positive results, and since there is always a risk that it does not, re-randomisation is simply too expensive to consider, even if it would be scientifically the best way to go about the problem of controlling mid-trial for unknown confounders. In actual clinical practice the least time- and money consuming solution to the problem is to factor the now-known confounders in post-trial. The method most often used to account for confounders after a trial is the 'analysis of covariance method' also called ANCOVA. ${ }^{84}$. However, ANCOVA only works reliably if the groups are homogenous. The method however does not work in every case. Sometimes it can only demonstrate the problem. And the same is true for

84 Medcalc. Easy to use statistical software. Software provider for statistical methods in medical research. https://www.medcalc.org/manual/analysis-of-covariance.php. Last accessed on January $23^{\text {rd }}, 2020$. 
almost all statistical models in use today. ${ }^{85}$ Therefore it is important that randomisation works in the first place without having to result to statistical means after the trial.

It is a simple mathematical truth however that very large and diverse trials are the ones which control the best for most possible confounders, since there are simply enough participants to equal out the groups. ${ }^{86}$ In small trials, and many trials are small, which means too small for randomisation to do its entire magic, confounders are, almost necessarily, unequally distributed, even if some sort of stratification has taken place. This is also the main argument John Worrall uses against randomisation. However, as Jeremy Howick rightly points out, the supporters of EBM and randomisation do not make that strong a claim that randomisation is the only control. Randomisation is an important tool in the set-up of trials, but not the only one to maintain internal validity. And many critics, including Worrall, do not provide a sufficient alternative to randomisation, save then saying that confounders can be controlled for statistically once a trial is finished. ${ }^{87}$

\subsubsection{Blinding - its problems and its virtues}

Blinding is used to control for many forms of bias, many of which can severely alter the results of every trial. Different possible biases are:

1. selection bias: the allocation of participants is done depending on their possible receptiveness to the treatment. Allocation concealment is therefore necessary and can be achieved through blinding.

2. performance bias: participants and personnel can both influence the results of the treatment if they know what treatment they are receiving. At least those two groups need to be blinded to avoid performance bias.

3. detection bias: Results can be interpreted in certain ways and made to look a certain way if and when the 'outcome assessor' is unblinded and knows if the novel treatment or the standard is to be assessed.

4. reporting bias: cannot be controlled for through blinding, but only through rigorous enforcement of the rule that all research findings need to be published, the positive as well as the negative ones. Preferably even the raw data is published, or at least easily accessible for researchers.

85 K. P. Suresh. (2011): 8-11.

86 Jeremy Howick. (2011): 50. Howick and many others have already pointed that out. It is almost impossible to create a trial that is sufficiently large enough to control for all confounders. That is why in the set-up for most trials, in-trial check-ups are mandatory to see if the distribution is still equal.

87 John Worrall. (2003): 
Many authors have defined more biases, but these four are the most important ones because they are the ones that have to the most influence on the actual trial results.

Randomisation without blinding can only control for the initial selection-bias if and when the researcher is prevented through randomisation to decide to which treatment arm the participant is allocated. ${ }^{88}$ All further forms of selection-bias and especially the selection-bias on part of the participant are only avoided if at least the researcher and the participant are blinded. "Six groups involved in a trial are sometimes blinded, namely participants, caregivers, data collectors, outcome evaluators, statisticians, and manuscript authors." ${ }^{89}$ However it is often not defined or explained in research protocols and post-trial write-ups which of the six possible groups was actually blinded. ${ }^{90}$ All groups can be filled in by different people or one person can fill in almost all of the roles. In the write-up of an RCT it should be made clear not only which groups were blinded, but also how many different people or groups there were to blind, and if the blinding could be maintained for the duration of the trial.

Howick for example groups together the researchers and those who are dispensing the treatment into "caregivers" but separately names "data collectors" and "outcome evaluators." It seems to be an arbitrary decision and one that every author and researcher can make for themselves. The lack of a reporting standard and the lack of clear definitions of terms can make the results of RCTs less robust and therefore vulnerable to manipulation.

The most often used method of blinding is double-blinding. This means that at least participants and researcher are blinded. If Howick's terminology is assumed, researcher means caregiver. 'Caregiver' can include the acting physician and possibly the nurse who might dispense the treatment. In order to successfully maintain the blinding however it should be at least triple-blinding, including the participants, those who dispense the medication and the researcher. The latter should be blinded regardless if he or she is having an active part in the trial, or is more on the sidelines, organising the trial and assessing the results.

After a blinded randomisation, blinding has to be maintained during the trial. The randomisation codes should not be revealed and all possible treatments, be it the new, the standard, or placebo has to be sufficiently alike to not be distinguishable from each other. There are different methods available to achieve this, depending on the type of the treatment. Oral treatments, as in pills or liquids, can be manufactured in a way that the control treatment looks, smells and tastes exactly like the treatment under test. This is true for both placebo-controlled trials and

88 John Worall. (2004): 2.

89 Jeremy Howick. (2011): 65.

90 Isabelle Boutron and David Sackett, et.al. (2006). "Methods of Blinding in Reports of Randomized Controlled Trials Assessing Pharmacologic Treatments: A Systematic Review." PLoS Medicine, Volume3, Issue 10: 1923. 
active controlled trials, where the novel treatment is tested against the accepted standard. Taste and smell can be masked by strong flavours such as peppermint or simply sugar. Food colouring works as well, or the use of gelatine capsules which all look the same from the outside and have the same texture. ${ }^{91}$ There are many methods available to make the different treatments, standard, novel and placebo to look and taste alike and the whole scope of possibilities is usually used to successfully blind all those who are involved in the trial. Treatments which have to be administered intravenously or intramuscular or any other way rather than oral, can also be manipulated in such a way as to mimic the treatment under test.

It can be hard to maintain the blinding, especially in cases where the intervention under test yields dramatic results, either positive or negative, very early on. For some philosophers of science dealing with EBM such as John Worrall or Jeremy Howick, the fact that the blinding can be hard to maintain and easy to subvert is a real problem. However, I believe that especially in those cases were there are dramatic results, the occurring unblinding can be easily dealt with. And sometimes it is even necessary to quickly and effectively treat the participants, especially when the effects are dramatic and not in a positive, but in a negative way, as for example in the TGN1412 trial of $2006 .{ }^{92}$ In the TGN1412 phase I trial, eight healthy male participants were randomised to receive either TGN1412 or a placebo. Six received the active treatment and deteriorated very quickly due to a cytokine storm, a condition in which the entire immune system and consequently the organs are shutting down very quickly. ${ }^{93}$ Accordingly it was immediately obvious which two participants had received the placebo. All six participants who had received the active treatment were subsequently treated in intensive care and survived, but they did so with lasting repercussions to their health and well-being. The most dangerous 'flaw' that happened during the trial was that the initial dose was too high and that the intervals in which the drug was administered were too short. The waiting period between the single administrations of the drug in each individual participant and the intervals between administering the drug to the next participants should have been significantly longer. ${ }^{94}$

However, it is important to realise that especially phase I trials have the potential to be dangerous for the participants and are therefore very tightly controlled. They are only be approved if a maximum of safety can be guaranteed. The unblinding in the example occurred quickly and without the possibility of avoiding it, much to the safety and security of the participants who could be treated fairly quickly.

91 Isabelle Boutron and David Sackett. (2006): 1935.

92 Michael Goodyear. (2006). "Learning from the TGN1412 trial." in BMJ: 1-2.

93 E. William St. Clair. (2008). "The calm after the cytokine storm: lessons from the TGN1412 trial" in Journal of Clinical Investigation. 118(4): 1344-1347.

94 E. William St. Clair. (2008): 1344-1347. 
A further problem which Howick mentions in connection to blinding is the Philip's paradox which claims that many dramatically successful interventions are not supported by best evidence, if one follows the claim that best evidence can only be attained through successfully blinded and randomised RCTs. ${ }^{95}$ The usual examples for such interventions are the Heimlich manoeuvre to unblock a closed airway of a chocking person or the removal of the appendix in a patient with acute appendicitis. However, it seems as if the Philip's paradox is not a real problem for medical research. Nobody would propose a non-surgical option for acute appendicitis and similar surgeries. It sometimes seems as if EBM proponents claim that research always starts at the bottom and has to work its way up. Instead, $76-96 \%$ of treatments used today are actually proven to be effective and can be used as comparison, or base, for novel treatments. ${ }^{96}$ And since it is less ethically questionable to compare a novel treatment against the standard treatment, instead of against a placebo, it is actually of great value that the available 'standard' treatments are that effective.

\subsubsection{Blinding in surgical trials}

Surgical trials are a special area of medical testing, because some important tools that make RCTs so internally valid are difficult to maintain during a surgery. Blinding would be one of these tools. Among all the groups which can possibly be blinded, at least the performing surgeon needs to know which surgery he or she is performing. ${ }^{97}$ There are two surgical trial options available however in which at least the patients and most other groups can be blinded. Either the novel surgery is compared against the standard form of surgery, or it is compared against a form of 'placebo' surgery. The latter is a so-called 'sham' surgery. 'Sham' surgeries mean that the patient is prepped as for a real surgery. A small incision is made and subsequently looks like a real wound, just that it is not as deep and the resulting scar might be less dramatic. Since the patient still has to receive anaesthesia, there is a certain risk of complications involved without any benefit at all, especially when 'general anaesthesia' is used. And even small incisions can lead to scars and sensitive tissue around the surgical area and hence, in the worst case, to longterm problems. So there is, even when sham surgeries are used, no entirely safe way to perform surgical trials. These trials are often performed with the overall goal to make a surgery as minimally invasive as possible or to reduce the time and amount

95 Jeremy Howick. (2011): 64.

96 Jeremy Howick. (2009). "Questioning the Methodological Superiority of 'Placebo' over 'Active' Controlled Trials." in The American Journal of Bioethics, 9(9): 33-48.

97 Paul Karanicolas, Forough Farrokhyar, and Mohit Bhandari. (2010). "Blinding: Who, what, when, why, how?" in Canadian Journal for Surgery, 53(5): 345-348. 
of anaesthesia. Anaesthesia itself is very risky and can even be life-threatening. Recently researchers are focusing more on the connections between anaesthesia and amnesia and even dementia. ${ }^{98}$ Anaesthesia for major surgery can, and often does, lead to to some type of temporary memory loss. Most patients experience that memory loss but do not suffer any further consequences because it is such a short-term phenomenon. In rare cases when the patient wakes up during surgery, the intrinsic memories of waking up, irrelevant if the patient actively remembers or not, can lead to post traumatic stress disorder and therefore to longterm harm for the patient. ${ }^{99}$ Current research also investigates the risk of dementia caused by anaesthesia for older, not necessarily only elderly, patients. So far there are not enough research results to back-up the hypothesis of a connection, but that does not mean that there is no risk. ${ }^{100}$

The use of 'sham' surgeries is also regulated by the Declaration of Helsinki, since 'sham' surgeries are equal to the use of placebo, albeit having a higher risk. The Declaration of Helsinki states that the use of placebo is allowable "where for compelling and scientifically sound methodological reasons the use of any intervention less effective than the best proven one, the use of placebo, or no intervention is necessary to determine the efficacy or safety of an intervention and the patients who receive any intervention less effective than the best proven one, placebo, or no intervention will not be subjected to additional risks of serious or irreversible harm as a result of not receiving the best proven intervention. Extreme care must be taken to avoid abuse of this option. "101 (My emphasis). Following the Declaration of Helsinki, it is advisable to compare new surgical methods against the standard. That the blinding here cannot be fully maintained has to be accepted.

\subsection{Placebo and the placebo response}

Placebo means in Latin "I will please". Placebos are pills or injections that look and smell like a treatment with an active ingredient but are actually made either from sucrose or lactose or come in the form of a saline solution as an injection.

98 V. Fodale and L.B. Santamaria, et.al. (2010). "Anaesthetics and postoperative cognitive dysfunction: a pathological mechanism mimicking Alzheimer's disease.” in Anaesthesia, 65: 388395 .

99 Walter Glannon. (2014). "Anaesthesia, amnesia and harm." in Journal of Medical Ethics. 0:1-7.

100 Alzheimer's Research UK. "General anaesthesia linked to increased dementia risk." Published online May 31st, 2013. http://www.alzheimersresearchuk.org/general-anaesthesia-linked-to-increased-dementia-risk/. Last accessed October 15th, 2017.

101 World Medical Association, The. Declaration of Helsinki. Adopted by the 18th WMA General Assembly, Helsinki, Finland, June 1964. Lastly editorially revised by the 64th WMA General Assembly, Fortaleza, Brazil, October 2013. Article 33, Use of Placebo. 
Placebo pills can be manufactured and manipulated in many ways to mimic an actual treatment, but usually do not contain any active ingredients. Placebos can however trigger a special effect in the patient, despite their lack of active ingredients. "The placebo effect arises out of the patient's confidence in the treatment (of the physician)." 102 It can be triggered by the physician, if he or she can convince the patient that the offered treatment will be succesful. These instances of the use of the placebo-effect are linked to the phenomenon of 'classical conditioning' and refer back to the physiologist Ivan Pavlov who discovered that his laboratory dogs would salivate if he, or one of his assistants, would enter the room to feed the dogs. After a while they would salivate even if one or the other would arrive without food. The dogs were conditioned to react in that way, regardless of the presence or absence of food. In the same way a human being can be conditioned to believe in an effect, even if the there is no actual effect to be measured, as in the use of placebo. ${ }^{103}$

The American anaesthetist Henry Beecher used saline injections on soldiers during World War I when morphine was running low. He discovered that they worked, especially when he told the soldiers that they received a powerful pain medication. ${ }^{104}$ Again, the placebo-response here refers back to some type of conditioning which can be very strong. In the instance of the soldiers receiving the injection, the use of placebo was ethically correct because it had, due to the conditioning, a positive effect on the patient and there was no active treatment available. However, successful placebo effects can lure the patient into a false sense of security, since only the symptoms are addressed, not the underlying disease.

Testing active treatments against placebo factors the placebo effect for both into the results. Every active medication contains both, the active effect and the placebo effect. ${ }^{105}$ However the placebo effect is not present in every patient and it is certainly not the same for every patient. ${ }^{106}$ Therefore it would be a logical fallacy to attribute a $100 \%$ occurrence to the placebo effect. Still, it needs to be taken into account. When a new active ingredient is tested against the standard treatment, the placebo effect is also factored in, because it is potentially present in both cases.

Since the placebo effect is unspecific and can vary from patient to patient, it is difficult to filter it out for correct assessment. Some participants do not react at all to the placebo, some might react overly strong. And there is always the possi-

102 Edzard Ernst and Simon Singh. (2008):57.

103 Ivan P. Pavlov. (2003). Conditioned Reflexes. New York: Dover Publications. [Book is a compilation of his writings over time.]

104 Henry K. Beecher. (1955). "The Powerful Placebo.” in JAMA: 1602-1606.

105 Edzard Ernst and Simon Singh. (2008): 65.

106 Natalie Grams. (2015). Homöopathie neu gedacht. Was Patienten wirklich hilft. Berlin, Heidelberg: Springer Verlag: 133. 
bility that the actual placebo effect is confused with other naturally occurring effects of the disease. Regression to the mean or fluctuation of symptoms can be mistaken for, and reported as, a placebo-effect, without the possibility of clarifying which is which. ${ }^{107}$

\subsubsection{Passive versus active placebo}

To make matters even more complex, placebos can come in two different forms, active placebo and inactive placebo. Inactive or pure placebos are those that mimic the active treatment in looks, smell and taste but contain no active ingredients. Active placebos are supposed to mimic all of the above and additionally contain active ingredients to bring only the side-effects about. ${ }^{108}$ If the side-effects are known or suspected beforehand and can be included in the placebo, then in the case of positive side-effects the inclusion might be at best beneficial and at worst without any consequences at all, since not all possible side-effects have to appear in every patient. The case is different with regard to negative side-effects. In some treatments, the negative side-effects are well known and accepted because the overall outcome of the treatment is beneficial and the negative side-effects are just temporary. Chemotherapy is an example in which harmful side-effects are taken for granted, but are accepted because the benefits outweigh the harms done by the treatment. The same is true for every surgery, in which bodily harm is inflicted for the purpose of healing the patient.

To produce a placebo with harmful side-effects, but without any positive treatment effects, can potentially pose a real risk to the health of the participant that is not outweighed by some positive outcome. These placebos are also called nocebos and they can either be manufactured or the nocebo-effect appears in the patient, although an inactive or an active placebo has been given. ${ }^{109}$ "Nocebo" again is latin for 'I will harm.' Since risks should be minimised in trial settings, active placebos are highly problematic and their use is ethically challengeable. In cases where an active placebo would be needed, because otherwise the blinding is impossible to maintain due to the lack of side-effects in the control arm, it would be advisable to opt for the standard treatment as a possible control instead. Only if there is no standard treatment available should an active placebo be used.

107 Damien G Finniss, Ted J Kaptchuk, Franklin Miller, Fabrizio Benedetti. (2010). "Biological, clinical, and ethical advances of placebo effects." in The Lancet; 357: 686-95

108 Isabelle Boutron and David Sackett. (2006): 1938.

109 Herbert Benson. (1997). "The Nocebo Effect: History and Physiology." in Preventive Medicine, 26 (5): 612-615. 
However, even inactive placebos are not without problems. They might suffer from a misnomer inasmuch as that there is no such thing as an inactive pill. Placebos contain something and they trigger a response, and even if it is just the bodies response to a small dose of sugar. Can something that does something ever be called inactive? ${ }^{110}$

Some proponents of placebo-controlled trials are adamant that the side-effects should be prevalent in the placebo, since it would be impossible to keep the blinding if the treatment arm noted side-effects and the control arm does not. ${ }^{111}$ Again, due to the placebo effect however, participants in the placebo arm might report side-effects. It is not as clear-cut a phenomenon as some of the placebo-trial proponents like it to be. The knowledge and subsequent sharing of any side-effects can lead to contamination and can therefore invalidate the results of the trial. The question remains if the risk of contaminating the results is really higher than the risks inherent to the actual side-effects of an active placebo? The reason why I estimate the risk of the active placebo higher is that it is a direct risk to the participant and one which cannot be avoided if active placebos are used. The risk of contamination can be avoided by separating the participants of the two treatment arms whenever it is possible. In other cases, when participants are bound to meet in the clinic or the doctor's office, what I would call patient to patient contamination, could be avoided by putting a clause in the initial agreement that forbids to talk about any symptoms of the medication. Allegedly that approach does not help against possible selection bias on the part of the researcher when the obvious absence of known side-effects jeopardises or even violates the blinding. However, a trial can still yield successful results, even if the blinding is terminated prematurely, as in fact happens in many trials. ${ }^{112}$

\subsection{Stopping clauses}

The 'ideal' running of a trial would entail that it runs until set endpoints are reached. The timing is often pre-calculated, since trials are expensive and cannot run indefinitely, due to lack of funds and willing participants. As we have seen before, trials are run to either test for safety or efficacy or both. Every trial however can be stopped prematurely, if and when necessary, as was for example the TGN1412 trial. In order to do so, many trials have stopping clauses implemented in their protocol. Stopping clauses define when a trial can be stopped for either safety or efficacy, before it actually has run its pre-ordained course. In order to

110 Jeremy Howick. (2011): 72.

111 Jeremy Howick. (2009): 37. And Howard Brody and Franklin Miller. (2002). "What makes placebo-controlled trials unethical?" in American Journal of Bioethics. 2(2): 3-9.

112 Jeremy Howick. (2011): 
successfully use stopping clauses however, terms like 'harm', 'safety' and 'efficacy' must be scrutinised closer.

Safety and efficacy are both natural endpoints for trials; as are mortality and morbidity. The TGN1412 trial is one example where the trial was stopped prematurely because of harm to the participants. Harm is divided into toxicity and death. In the literature the terms 'harm', 'safety' and 'toxicity' are often used interchangeably. "...morbidity and/or mortality outcomes may reflect both risk and benefit. ${ }^{113}$ As counterintuitive as this sounds, there are cases where obviously contradicting endpoints where established and one was deemed to be the actual endpoint, while the other was supportive, or surrogate, endpoint. This distinction means that negative results can be measured in interim analyses and do not lead to the trial being stopped, because they are considered to be a normal part of the trial. But one which is only limited and still supports an overall positive outcome. The idea behind this utilitarian sounding reasoning is that it cannot be quite clear if a treatment is harmful or successful until all the data is accrued. Surrogate endpoints should never be used as a reason to stop a trial prematurely. ${ }^{114}$

There are four specific reasons that are mentioned in most of the literature for stopping a trial early: ${ }^{115}$

1. adverse effects leading to unacceptable safety hazards

2. futilitiy, that means that the sample size is too small to achieve significant values

3. new external information that either answers the question or raises safety issues

4. apparent benefit

Following Bassler's et al. reasoning here, it would be best if interim analysis are done by an independent committee. These committees are data monitoring committees (DMC) or data safety and monitoring boards (DSMB). They should be independent from those organising the trial and are therefore not blinded. If a committee is part of a trial set up, it can be blinded, because it would be sufficient to have it see the raw data. That is another reason why these committees are used more and more frequently. They can act as outside observers who do not have any interest in the outcome of the trial and who can therefore more easily determine if there is a problem in the setup or the conducting of the trial. "Nowadays, some

113 Yanli Zhao, Patricia Grambsch and James D. Neaton. (2007). "A decision rule for sequential monitoring of clinical trials with a primary and supportive outcome." in Clinical Trials, (4): 140-153.

114 Jan Sprenger and Jacob Stegenga. (2017). "Three Arguments for Absolute Outcome Measures." in PSA2016: The 25th Biennial Meeting of the Philosophy of Science Association.

115 Dirk Bassler, Victor M. Montori et. al. (2008). "Early stopping of randomised clinical trials for overt efficacy is problematic." in Journal of Clinical Epidemiology 61: 241-246. 
funding, ethics, and regulatory bodies consider an independent DMC essential for major RCT's. For instance, the Food and Drug Administration has published a draft guidance for clinical trials sponsors on the establishment and operation of clinical trials DMCs." ${ }^{116},{ }^{117}$ The important note on the FDA guidance is that it is non-binding. That means that other guidelines can be used, if and when the FDA ones do not fit to the actual setup of a trial. On the one hand, this approach gives room for multiple ways of setting up of trials and of establishing stopping clauses and DMC's, on the other hand, non-binding guidelines mean that they do not have to be used at all, and that trials can be set up without these safeguards.

If a trial is set up with stopping rules, there are initially two ways in which those can be used. One way is an 'or' stopping rule, which means that the trial is stopped "for either a safety or efficacy outcome." 118 The other way is an 'and' stopping rule, meaning that the trial is stopped for safety and efficacy, rendering the results potentially more robust. ${ }^{119}$ In phase 0 and I trials, 'safety' is the main concern of the trial and therefore the natural endpoint. In phase II, III and IV trials, when a base-safety is established, 'efficacy' is of higher concern and can be the more natural endpoint of a trial, without neglecting safety. If real safety concerns do appear in later trials, those are definitive indicators to potentially stop the trial prematurely.

There are multiple ways, and multiple reasons, to terminate a trial before it would actually end. Stopping rules should be decided before the start of a trial and specified in the trail protocol.

Judged by the amount of literature about stopping rules in the case of benefit (a lot), ${ }^{120}$ compared to the case of harm (very little) ${ }^{121}$ it would seem as if the decision to stop a trial in case of harm or toxicity is fairly easy. However, if looked at it closely, even in the case of harm, it is not an easy decision to stop a trial. A multitude of concerns can play a role here. Ethically it sounds obvious to stop a trial that is dangerous for the participants involved. However, some 'harms', like some side-effects might be only temporary. "The decision to stop early for harm is potentially more complex than benefit or futility because it may involve a trade-

116 Yanli Zhao, et.al. (2007): 142.

117 Food and Drug Administration (FDA) guidelines: "Guidelines for clinical trial sponsors." https:/www.fda.gov/downloads/regulatoryinformation/guidances/ucm127073.pdf. The guidelines are fairly specific but nonbinding. It is assumed that when a different method is more appropriate for the test on hand then that method will be used. Last accessed on January $23^{\text {rd }}, 2020$.

118 Yanli Zhao, et. al. (2007): 140.

119 Yanli Zhao, et.al. (2007): 141.

120 Unrefined PubMed search with the search terms "stopping rules due to benefit" yielded 67 results.https://www.ncbi.nlm.nih.gov/pubmed/?term=Stopping+rules+due+to+benefit. Last accessed on January $23^{\text {rd }}, 2020$.

121 Unrefined PubMed search with the search terms "stopping rules due to harm" yielded 8 results. https://www.ncbi.nlm.nih.gov/pubmed/?term=Stopping+rules+due+to+harm. Last accessed on January $23^{\text {rd }}, 2020$. 
off between potential - but as yet undemonstrated - benefits and apparent (but possibly spurious) adverse effects." 122 Mill et.al. argue further on how important these DMCs in the possible case of stopping a trial for harm are, because they might be able to distinguish real harm from side-effects and can react quickly to avoid greater harm for the participants.

In the case of stopping a trial for benefit, the question can only be if the benefit is a real benefit and not a spurious event at that particular point in the trial which makes the treatment look to be beneficial, but hides harms that would have come to light later on, for example through 'regression to the mean' of the disease in question. "The social value of the research is severely compromised when overly sanguine estimates of treatment effect result in misleading risk - benefit ratios, misguided practice recommendations, and suboptimal clinical practice...."123

Stopping clauses are important, because they can act as a safe-guard to prevent harm for the participant and in very rare cases they can speed up a drugapproval process, if, and only if, benefit is established beyond a doubt. The latter case can be important if and when no standard treatment is available and the treatment under test is the only one to prevent harm for the patients in the actual target population. This is most often the case in drug-trials concerning orphan drugs. ${ }^{124}$ Orphan drugs are those drugs that are treating a very small patient population with a very rare disease. Often orphan drugs are poorly researched because they bring little to no profit. However, if they are researched, most patients are quite desperate for the trial results because no other treatments are available. If early on a benefit is detected it might be possible to stop a trial early and launch a phase IV trial after market approval. Another solution however would be to enter in a compassionate use program, so that patients outside the trial can receive the treatment and the actual trial can run its pre-considered course, without the recourse to any stopping clauses. $^{125}$

\subsection{External validity}

As we have seen, when RCTs are performed correctly, they are internally valid and therefore their results should be robust and presentable. Questions however remain: presentable for what, and for whom? Can the treatment be used in the

122 Edward Mills, Cooper Curtis, et.al. (2006). "Randomized Trials Stopped Early for Harm in HIV/AIDS: A Systematic Survey." in HIV Clinical Trials 7(1): 24-33.

123 Dirk Bassler and Victor M. Montori. (2008): 244.

124 Aaron S. Kesselheim, Jessica A. Myers and Jerry Avorn. (2011). "Characteristics of Clinical Trials to Support Approval of Orphan vs Nonorphan Drugs for Cancer." in JAMA, 305 (22): 2320-2326.

125 Hanna I. Hyry and Jeremy Manuel et.al. (2015). "Compassionate use of orphan drugs." in Orphanet Journal of Rare Diseases. 10 (100). 
target population as it is used in the treatment population? Is the treatment even feasible in the target population? How can the problem of external validity or extrapolation, as it is also called, be solved?

The results of a RCT with a positive outcome show us that the treatment can be used for the population in which the treatment has been tested. However, treatments are not developed for some small trial population, they are supposed to work in the wider population who suffers from this specific disease. But are the treatment and the target population sufficiently alike so that the treatment could be successfully used in the actual target population? How can this necessary 'alikeness' be established? The problem of transporting research results from trial conditions to the actual target population is called the problem of external validity or of extrapolation. Some authors do use the term generalisability, but for the sake of clarity, I will stick with 'external validity', as the opposite to 'internal validity.' Howick prefers to use the term extrapolation, because sometimes it can be necessary to extrapolate results within a trial to make it applicable to a participating subgroup. As an example Howick uses a trial in which a beneficial result was only pertaining to a very small subpopulation, within the larger trial population, with a very specific condition. ${ }^{126}$ Even though the results have been extrapolated to a specific target population, the trial over all is not at that point deemed to be externally valid.

It is often said that the higher the internal validity of a trial, the bigger the problem of external validity. So the focus here is on assessing what contributes to the difficulty in extrapolating results in the set-up of, and recruitment for, RCTs and how trials results can be made usable for evidence-based practice.

\subsubsection{External validity and trial design}

The problem of external validity already becomes prevalent in the set-up of a trial. The claim: 'the more internally valid a trial is, the less externally valid the results seem to be' is already precluding that the 'ideal' set-up of a trial might not be the 'best' set-up. ${ }^{127}$

The randomisation process in RCTs is used to prevent selection bias and to render control-and treatment arms as alike as possible, in order to achieve a common base line. Participants are chosen in such a way as to accomplish comparability. A certain similarity is therefore helpful. Most trial designs include a run-in

126 Jeremy Howick, et.al. (2013). "Problems with using mechanisms to solve the problem of extrapolation." in Theoretical Medicine of Bioethics; 34: 277.

127 Nancy Cartwright. (2007). “Are RCTs the Gold Standard?” in BioSocieties. (2): 11-20. 
period as a so-called 'enrichment strategy'. ${ }^{128}$ One type of run-in period is when already included participants have to submit to a wash-out period before data is gathered. That means that they have to stop taking other drugs or stop certain habits, like smoking, so that no traces are left in the body, before they can participate in the trial. A different form of wash-out period is when participants, also before data gathering, are given placebos and those participants that respond to the placebo are not included in the actual trial. ${ }^{129}$ Reaction to placebo however can again be very varied and might be hard to detect, since any change in the patient receiving the placebo can have reasons unrelated to the non-working treatment. It is questionable therefore if a run-in period based on the placebo criteria is helpful.

Another type of run-in period is the randomised withdrawal design. Here participants are excluded from the trial when they are either non-compliant or if they show toxic effects because of the treatment under test. All types of enrichment strategies are compromising the external validity of RCTs. But they are still fairly prevalent, because they guarantee a more favourable result overall, and the more favourable the result for a new treatment, the earlier it will be approved and released into the market, thereby making profit. Hence, any form of enrichment strategy of a trial should be avoided if the trial results are supposed to be external valid overall and valid for the individual patient as well.

When all possible participants are included in the trial, with or without a runin period, then the researchers have achieved nearly perfect laboratory conditions. But they have also narrowed the playing field considerably. The ideal participants for a trial seem to be male, in their mid-thirties to mid forties and without any coor multi-morbidities. ${ }^{130}$ And even if gender and age is more mixed, the lack of coand multi-morbidities plays a significant role, because they can severely influence the outcome of any trial. Either because necessary medication that cannot be flushed out, interacts with the novel treatment or the disease under trial is overshadowed by these other diseases. Female patients are asked less often to participate in a trial, even if the treatment in question is treating a disease that is not dependent on gender. ${ }^{131}$

Obviously most trial participants in real life are far removed from the above ideal. The chief reason for this is that almost no one, regardless of gender, is free

128 Robert Temple. (2012). "Enrichment Strategies." in Guidelines of the U.S. Food and Drug Administration. https://www.fda.gov/ucm/groups/fdagov-public/@ffdagov-afda-orgs/documents/document/ucm 303485.pdf. Last accessed on September 15th, 2017.

129 Ariel Pablos-Méndez, R. Graham Barr, and Steven Shea. (1998). "Run-in Periods in Randomized Trials Implications for the Application of Results in Clinical Practice." in JAMA 279(3): 222-225.

130 Ben Goldacre. (2012): 159.

131 Wendy Rogers. (2004). "Evidence-Based Medicine and Women: Do the Principles and Practice of EBM further Women's health?" in Bioethics. (81)1: 61. 
of co - or multi-morbidities. Simple examples for this can be asthma, skin or joint problems, etc. Those minor ailments which the patient does not even perceive as morbidities but which nonetheless can alter the outcome of a trial can and are used to exclude possible participants from trials. Hence, the wish to either exclude as many of these predicaments or to distribute them equally to both groups is very understandable in theory, but hard to maintain in practice. Equal distribution is a valid goal in trial designs, but the exclusion of possible participants based on their co-or multi-morbidities is not advisable, since the results that would be garnered by such an idealised trial would not be generalisable to the target population. Often age does play a role. Children and the elderly are often excluded, again explained as a precaution. "Up to $90 \%$ of potentially eligible participants are sometimes excluded from trials according to often poorly reported and even haphazard criteria. For example, the most effective antidepressants in adults have doubtful effects in children." $" 132$

\subsubsection{External validity and recruitment}

Since the ideal participant, with the qualities outlined above, seems to be nonexistent, the goal the recruiter has to aim for is 'eligible'. The patient has to be eligible to become a participant. Therefore, recruitment of a group of eligible patients is of the utmost importance for a trial. But who is eligible? First of all, the patient/participant has to have the illness in question to even be considered for a trial from phase II onward. Phase I is usually conducted with healthy volunteers. ${ }^{133}$ Secondly, the patient has to be at the right place at the right time. Some trials are advertised openly, but recruitment often happens on a walk-in basis, meaning that the clinic conducting the trial asks those patients who are walking in with the disease in question, to participate. Naturally, that excludes many eligible patients who are treated in a different clinic or by a GP who is not informed about the trial. Open advertising does not reach that many possible participants because as always one has to be attuned to look out for it. If one is not aware about the possibilities of a trial and is not made aware by their health-care provider, the clinics miss out on many eligible volunteers.

132 Jeremy Howick, et. al. (2013): 277.

133 There are, as always, exceptions to this rule. Some phase I trials are conducted in participants with the illness in question, because the treatment is so special that it should not or could not be trialled in healthy volunteers. A currently running trial at the UKE in Hamburg comes to mind in which children with NCL2, a severe form of childhood dementia, are receiving a very experimental treatment that would not be ethical to give to healthy volunteers. https://clinicaltrials.gov/ct $2 / \mathrm{resu}$ lts cond $=$ NCL2\&term $=\&$ cntry $=\&$ state $=\&$ city $=\&$ dist $=$. Last accessed on January $23^{\text {rd }}, 2020$. 
Even if the patient is eligible and is asked to participate, he or she might have good reasons to refuse. Many patients are afraid of being treated as human guinea pigs and rather opt for the standard treatment. Or they are afraid of just receiving the placebo. However, if they agree, it can be for many different reasons which subsequently can influence the actual outcome of the trial.

There are different types of patients which are participating in trials, but almost none do so for a greater societal good. Either there is a financial component involved, or there is a real sheer need on the part of the patient, because the disease is so rare or so little studied, that even the chance of receiving some treatment, $50 / 50$ in placebo controlled trials, is better than receiving none. A financial component can be that the patients are paid, as is very common in phase I trials where healthy participants are recruited. Again the TGN1412 trial comes to mind. And even in later trials, some sort of compensation is often offered. ${ }^{134}$ Another case can be that patients within a trial receive care and medication for free and do not have to pay for it or have to claim payment from the insurers. Often trial medication can be very expensive. Additionally, in a trial setting, clinicians are a lot more thorough and the overall care is often better and the patients are more closely monitored. So, for many patients, being recruited to participate in a trial can be a win/win situation.

There is an ongoing reluctance to recruit female patients into medical trials, especially pregnant or lactating women. And to underscore the validity of my point here, one only has to look at the package insert of most established treatments. It is almost always stated that the medication has not been tested on pregnant and lactating women and should therefore not be taken by that individual group of patients. "Until 1993, the FDA excluded women of childbearing age from participating in early (Phase I and II) drug treatment trials, and this reduced their enrolment in Phase III trials (usually randomised controlled trials)." 135 It is easy to follow the actual motive behind that exclusion. Pregnant women are especially vulnerable and to not include them in any trials is often only done to safeguard them and the unborn child from harm. This became one of the cornerstones of trial recruitment after the thalidomide scandal. But as valid as this thinking is on a first glance, it is unfortunately not entirely feasible, even for pregnant women. An unfortunate consequence of this exclusion is that pregnant, and most of all, birthing women are subjected to all sorts of non-best-evidenced treatments. Among those are birthing positions, anaesthesia, and pain medication, to name the most common ones. These treatments and procedures have hardly changed over time. And some of them might be unnecessary or even harmful, but nobody challenges the practice

134 Christine Grady. (2005). "Payment of clinical research subjects." in Journal of Clinical Investigation. 115(7): 1681-1687.

135 Miriam Solomon. (2015): 142. 
since there is no good evidence to do so. Pregnant and lactating women are therefore a huge group of patients which is still to a large extend excluded from evidence-based research. However, these women still might need treatment for certain conditions or are even dependent on it, especially if they have chronic diseases, like heart diseases or certain forms of rheumatoid arthritis. In both cases, as in many others, in order to manage the disease next to the pregnancy, the woman has to continue the medication, even though the evidence to do so is only based on experience and not on the best evidence possible. ${ }^{136}$

It was generally assumed that research results based on research conducted with male patients could easily be extrapolated and used in a female target population. "The gender bias amongst participants in clinical trials is well known. Women have been excluded from research for many years, for a variety of reasons including the alleged need for homogenous populations, the fear of harms to pregnant women, the cost of including women, and the purported difficulty of recruiting women." ${ }^{137}$ It must be obvious to even those who have a limited medical knowledge that results cannot be easily transferred from one gender to the next. Although the core functions might be the same, even symptoms of the same disease, i.e. stroke, might display differently depending on the gender of the patient. ${ }^{138}$

Minorities are another group of patients who is consistently neglected in the research of EBM. In the United States, African Americans and Latinos are consistently underrepresented in medical research. Most often because they are afraid to be used as guinea pigs. ${ }^{139}$ It is often assumed that the experiences of Tuskegee, were only black males were recruited, without being told about their participation in a trial, are still prevalent in society and this being the reason why those minorities refuse to participate in research. ${ }^{140}$ Wendy Rogers argues against this, claiming that because of their specific health needs or set-up, they are simply not asked to participate and would do so, if there would be a possibility. Many minorities in the United States do not have health insurance or see a GP on a regular basis. Research might help them, but is as equally unavailable to these groups as is regular health care. "Given higher rates of morbidity and mortality among ethnic minorities in comparison with majority populations, this lack of representation in

136 Margaret A. Honein, Suzanne M. Gilboa, and Cheryl S. Broussard. (2013). "The Need for Safer Medication Use in Pregnancy" in Expert Review of Clinical Pharmacology. 6(5): 453-455.

137 Wendy Rogers. (2004): 11.

138 A.H.E.M. Maas and Y.E.A. Appelman. (2010). "Gender differences in coronary heart disease." in Netherlands Heart Journal. 18(12): 598-602.

139 B.R. Kennedy, C.C. Mathis and A.K. Woods. (2007). "African Americans and their distrust of the health care system: healthcare for diverse populations." in Journal of Cultural Diversity. 14(2):5660.

140 The Tuskegee experiment is explained at length in the chapter about informed consent. 
research exacerbates the existing vulnerability of minorities to poor health outcomes." ${ }^{141}$ And it makes it difficult to extrapolate the results of a trial conducted with Caucasian patients to African American patients, precisely because of the reasons mentioned in the quote.

The same holds true for patients with disabilities or psychiatric problems. Both groups of patients are considered minorities and are often not recruited. Either because they are not able to give informed consent or because they are also considered vulnerable. Again the reasoning is somewhat flawed, since research results garnered with ideal participants cannot be used as such for patients that have many co-morbidities and are already taking medication that has to interact with any new treatment. Especially when patients have to take psychotropic drugs on a continuous basis the interactions with other treatments have to be taken into account and carefully monitored. These interactions can and should be tested in advance, namely before the medication reaches market approval.

Another factor that should be taken into account when recruiting patients for a trial is the fact that people do change over time. They might develop some other disease and they do simply age. Worrall gives an example in which the trial population and the target population differed in age and how that affected the outcome.

"One example is the drug benoxaprofen (trade name: Opren), a nonsteroidal antiflammatory treatment for arthritis and musculo-skeletal pain. This passed RCTs (explicitly restricted to 18 to 65 year olds) with flying colours. It is however a fact that musculoskeletal pain predominantly afflicts the elderly. It turned out that, when the (on average older) 'target population' were given Opren, there were a significant number of deaths from hepato-renal failure and the drug was withdrawn." 142

The example not only shows that it can be dangerous to restrict the participants in an essential way. It also shows that if the existing clinical expertise would have been taken into account, the drug would from the beginning have been tested in an elderly population. The fact that "musculo-skeletal pain predominantly afflicts the elderly"143 was well known before the trial. The trial could have been both internally and externally valid if the right participants would have been recruited into it from the very beginning.

141 Wendy Rogers and M.M. Lange. (2013) "Rethinking the vulnerability of Minority Populations in Research." in American Journal of Public Health. 103(2): 2141-2146.

142 John Worrall. (2007). "Evidence in medicine and evidence-based medicine." in Philosophy Compass (2)6: 995.

143 John Worrall. (2007): 996. 


\subsubsection{External validity and n-of-1 trials}

To quote Jeremy Howick again "one type of randomised trial, namely n-of-1 trials have arguably the highest degree of external validity of any comparative clinical study." " 144 N-of-1 trials consist of one patient who receives either the treatment under test or a placebo or standard. In most cases the participant receives the placebo and the treatment on alternate weeks or month. Hence, the trial population and the target population is equal to each other.

$\mathrm{N}$-of-1 trials do sound like the perfect alternative to standard RCTs because of the guarantee of external validity, if internal validity is given. However, n-of-1 trials are less reliable than could be assumed on a first glance. Since they only involve one patient it can be impossible to ascertain if that patient has improved because of the treatment, or because of "spontaneous remission" or because of the placebo-effect. ${ }^{145}$ And it is impossible to infer how close this one patient resembles other patients with the same disease. N-of-1 trials are really only applicable for patients with chronic but otherwise stable diseases. Psoriasis and atopic eczema are examples for those, since a patient can test different skin treatments and see over time which one works the best. However, for most diseases, especially those which are unstable and are quickly changing, n-of- 1 trials are not feasible, because their results can not be extrapolated at all.

"For example, it is impossible to know whether aspirin will prevent a patient's stroke until it is too late. This is a problem with most cases of preventive medicine, and also with treatments for many acute conditions, such as meningitis, pneumonia or snake bite, where we don't have the opportunity to test it in each individual patient and see. So we then have to rely on whether and how to apply the evidence from the experience of studying others." 146

$\mathrm{N}$-of-1 trials are therefore no solution to the overall problem of external validity but only provide a solution in very exceptional cases.

\subsubsection{How can external validity be achieved?}

External validity is hard to achieve and is lacking as main goal in many trial designs. Nonetheless, external validity is what is needed to make the trial results applicable to the target population and preferably to the individual patient. One solution described above, but only in very special cases, can be N-of-1 trials, but they are seldom feasible. The most obvious solution must be proper recruitment

144 Jeremy Howick. (2011): 55.

145 Jeremy Howick. (2011): 55.

146 Jeremy Howick. (2011): 152. 
for trials. Since most people suffer from more than one condition, co-morbidities should not be discounted in the recruitment process but understood as known confounders and taken into account in the trial design. Women and children need to be included, in the case of children apparently only after careful consideration, into trial designs so that all age and gender groups are represented, depending on the disease and drug or treatment in question. That trials can carry a certain risk for the participant is acknowledged, but should not exclude the recruitment of all possible participants. In the next chapter I will discuss informed consent and the role it can possibly play in safeguarding participants as much as possible and how patients can be involved in the decision to participate.

Another way to make trial results more externally valid are phase IV trials. These happen after the market approval of a drug and can be either randomised trials or longitudinal observational studies. Goldacre proposes that, especially in cases where there are competing treatments for the same condition, large randomised phase IV trials should be conducted via a patient database to which every GP and clinician has access, at least in the UK. Germany has a fairly similar system. These databases are anonymising the data of the individual patient, but would provide the researcher with the overall number of patients having received a certain treatment and with the overall characteristics of these patients and observed adverse events or side-effects. Goldacre argues that when there is general uncertainty about which treatment is superior for a certain disease, the GP should use the regular prescription system, but instead of entering the patient data and printing a prescription, he would enter the patient into a randomised trial and either treatment A or B is assigned to the patient. The GP consequently reports in the follow-ups about the performance of the treatment and, over time, a patient population which benefits from either the one or the other treatment would be established. ${ }^{147}$ The follow-up, at least in the UK, would be very easy and would not entail any more work for the GP, since all patient-data is recorded by a computer system anyway. Apparently the only methodological virtue missing in this scenario is blinding. But Goldacre argues that since existing treatments are compared, the methodological role of blinding is not as significant, since patients often do not have a preference towards either treatment. And the negative effect of non-blinding is calculated against the overall long-time effect of such a study, which can in theory run indefinitely. In the proposed circumstances the number of patients/participants is definitely big enough to make a statistical difference, and the treatments are not tested in an idealised trial population, but in the actual target population. ${ }^{148}$ The problem of external validity is thereby solved. For a lot of diseases today there exists a standard treatment. New treatments are often either a variation of the standard or

147 Ben Goldacre. (2012): 227.

148 Ben Goldacre. (2012): 228. 
at least comparable to it. A phase IV trial as described above would however not work for placebo controlled trials. But with general uncertainty about the efficacy of a treatment and the possibility to compare 'new' against 'standard' in the normal patient population, these kind of phase IV trials would be highly ethical.

\subsubsection{Publication bias}

Publication bias, one of the biases that does not play a role for the internal validity of the trial, should also be eliminated to make the trial results externally valid. Only if all trial information and preferably even the raw data is available, can the results be assessed for the actual patient in an hospital or at the GP. Every missing piece of information, let alone unpublished trials that had negative outcomes, severely distort the evidence-base. ${ }^{149}$ All trials should be published, regardless of their outcome. This is what the AllTRials campaign is diligently working for across the globe. ${ }^{150}$ And the data should not be tampered with to make results look better. This kind of tampering is called 'spin' and severely distorts the data so that it looks favourable when in actuality it is not. ${ }^{151}$

A lot of trials today are sponsored by pharmaceutical companies. These companies are naturally interested in positive results for the drugs they are trying to bring to market approval. Negative results do not bring profit. So if a trial is negative, either the data is manhandled to a degree that it looks good, even if it would only be the case for a tiny sub-group of patients, or the data is not made public at all. As if the trial never existed. The most compelling reason why this approach to data-handling is unethical is that participants who have given their time and their health for research are not rewarded and their engagement was doubly in vain because another company, or researcher, might come up with the same, or a very similar, idea and conduct a trial, absolutely unaware that it already has been done and proved unsuccessful. As Imogen Evans and colleagues are saying: "Unnecessary research is a waste of time, effort, money, and other resources; it is also unethical and potentially harmful to patients." 152

149 Imogen Evans, Hazel Thornton, Iain Chalmers and Paul Glasziou. (2011): 163.

150 AllTRials Campaign. http://www.alltrials.net/. Last accessed on January 23 ${ }^{\text {rd }}, 2020$.

151 Carl Heneghan, Ben Goldacre and Kamal R. Mahtani. (2017). "Why clinical trial outcomes fail to translate into benefits for patients" in Trials. Bio Med Central: 1-7.

152 Imogen Evans, Hazel Thornton, Iain Chalmers and Paul Glasziou. (2011): 129. 


\subsubsection{Surrogate outcomes versus primary endpoints}

There are multiple ways in which data can be changed during and after the running of a trial. One method is to change the overall intended outcome in the middle of a trial. Outcomes need to be specified in the trial protocol. Changing them midtrial or after the trial, compromises the entire trial results, because the trial initially was not designed to look for these outcomes. Outcomes are often also called endpoints and are divided into surrogate endpoints and primary endpoints. A primary endpoint of a trial can be death. An often used example are trials for cardiac conditions due to high cholesterol. An obvious primary endpoint would be cardiac arrest and subsequent death of the patient. So a question like "how many patients need to be treated with a cholesterol lowering drug, in order to save one patient from pre-mature cardiac arrest and death?" might be a valid research question. However, specific causes of deaths are hard to come by in patients with a preexisting cardiac condition. Death might have occurred despite the successful lowering of the cholesterol. Or no deaths do occur in a given period, or they do not occur during the duration of the trial. Therefore it might be easier to opt for a surrogate, or soft, endpoint. In the example the measure of cholesterol in the blood. The argument would then be that lower cholesterol-levels overall lead to fewer overall cardiac arrests and therefore it is deemed sufficient to measure cholesterol levels. If cholesterol-levels are lower with the novel treatment than with the standard or placebo then the novel drug is deemed to be superior in preventing premature deaths due to heart attack. However, cholesterol levels, albeit playing a significant role in overall cardiac health, are not in themselves the cause of heart attacks, but are merely a contributing factor. ${ }^{153}$ And cholesterol levels can change during the day. They are to a certain degree susceptible to diet and exercise and are certainly not constant in any given patient over time. So a trial that chooses the lowering of cholesterol as a surrogate outcome for the efficacy of an overall heart medication does not represent any really valuable results for the actual patient. A good example often used in this context is the prescription of statins to patients with high cholesterol. Statins were considered to be the drug to lower cholesterol levels and to prevent heart attacks with a minimum of side-effects. ${ }^{154}$ Treatment with statins is a long-term treatment and patients were advised that if they needed statins, they would need them for the rest of their lives. New findings however have shown that statins do lower cholesterol, but do not lower the overall risk of a hear attack, which was the reason that they were prescribed in the first place. Since low or high cholesterol levels in the blood do not influence the overall well-being

153 JJ Kastelein, A. Wiegman, and E. de Groot. (2003). "Surrogate markers of atherosclerosis." in Atheroscleris Supplements. 4(1):31-6.

154 B. Ziaeian and G.C. Fonarow. (2017). "Statins and the Prevention of Heart Disease." in JAMA Cardiology;2(4):464. 
of the patient and are not detectable by the patient, statins do nothing for the everyday quality of life. If, on top of things, they do not prevent heart attacks, it is questionable if they are useful as a drug, especially on a long-term basis. The surrogate endpoint of lower cholesterol versus the primary endpoint of death due to cardiac arrest has not shown to be sufficient for the efficacy of the class of drugs that are called statins.

\subsubsection{Equipoise}

Clinical, and even personal, equipoise has been heralded as an ethical tool to decide if a RCT can be performed, and is neither superfluous nor posing a risk to possible participants. The principle of clinical equipoise simply means that there has to be genuine uncertainty on the part of the clinicians who are conducting a trial, whether or not a new treatment is really more beneficial than a conventional one for the same medical problem, before a clinical trial is started. Freedman, the one who coined the term 'equipoise' in the 1980's, argues that clinical equipoise is satisfied "if there is genuine uncertainty within the expert medical community - not necessarily on the part of the individual investigator - about the preferred treatment." ${ }^{155}$ Personal equipoise would mean that the individual investigator is in a state of uncertainty about which treatment is superior. However, personal equipoise is hard to maintain and even to establish in the first place. Therefore the focus of the debate is about clinical equipoise.

Worrall explains that the ethical dilemma is easily avoided, if one follows the rule that only RCTs are producing valuable evidential results, since before a RCT is conducted, there can be no certainty about superiority either way. The "telling" evidence about the novel treatment is missing. ${ }^{156}$ That presumes however that the only 'telling' evidence comes from RCTs. As has become obvious, and hopefully will become more so, not all 'good' evidence is automatically generated by RCTs. 'Good' evidence can come from experience and observational studies as well. The ECMO case will be used as an example in which there was good prior evidence that the treatment would be successful but where RCTs were performed because they were deemed necessary. Although clinical equipoise was already, and some even argue, severely compromised.

And what about clinical equipoise in placebo-controlled RCTs? Some argue that clinical equipoise is violated in placebo-controlled trials, since there cannot be a general uncertainty about which treatment is superior. The clinician therefore

155 Benjamin Freedman. (1987). "Equipoise and the Ethics of Clinical Research." in New England Journal of Medicine. (317): 141-145.

156 John Worrall. (2008): 8. 
would break the trust of the patient who believes to receive the best possible treatment. The distinction between research and clinical practice is important to answer that question. The patient who is participating in a trial has changed into a participant and the clinician has changed into a researcher. The participant has consented to being part of a trial and the relationship of trust has consequently shifted. Clinical equipoise here cannot be used as an ethical safe-guard for the patient. ${ }^{157}$ Nor is it a safe-guard in all other types of clinical trials. Again, clinical trials need to be performed before a novel treatment can achieve market approval. That is in most cases not only a technical but also a legal prerequisite. Therefore the question if it is legitimate to perform a RCT is raised far less often than Freedman and other proponents of clinical equipoise would like. And clinical equipoise is as easily subverted as is the blinding. If one treatment does better than the competitor, clinical equipoise is almost impossible to maintain. And again, patients agree to be part of a trial and become participants in research. The mutual trust between patient/clinician has shifted to participant/researcher and the expectation to receive the best possible treatment has changed to being part of a trial and not being subjected to unduly harm. ${ }^{158}$ Equipoise therefore does not seem to help in assessing the overall ethical questions of RCTs.

\subsection{Unnecessary trials}

Some trials are just unnecessary. For example trials which inadvertently are replicating trials which have already been performed but the results were, due to publication bias, never published. And one more ethical problem can be that RCTs are not necessary because the novel intervention is so convincing that a RCT would not have been required. Either the mechanistic evidence or quite simply the experience with the new treatment is so convincing that a RCT would do more harm than good, because participants really would receive the 'lesser' care when receiving the standard. Admittedly this later case is quite rare, and can only happen where non-drug treatments are concerned where there is no legal requirement to perform a RCT. The following example of such a case can be used to illustrate quite convincingly why it is not always in the patients interest, and overall better, to perform RCTs just for the sake of a statistical outcome. In the example the legal requirement to perform a RCT is also not relevant, since a procedure was put under test, rather than a drug.

157 Mario Castro. (2007). "Placebo versus Best-Available-Therapy Control Group in Clinical Trials for Pharmacologic Therapies. Which Is Better?" in Proceedings of the American Thoracic Society, 4(7): 570-573.

158 Anna Floyd and Anne Moyer. (2009). "Equipoise may be in the Eye of the Beholder." in The American Journal of Bioethics. 9(2): 21-22. 


\subsubsection{ECMO - or an example why not all RCTs are equal}

ECMO stands for 'extracorporeal membranous oxygenation.' The particular form of ECMO I want to discuss here was established to circumvent the lungs in neonates born with persistent pulmonary hypertension, a malfunction of the lungs, in which the lungs are not yet capable of oxygenating the blood sufficiently. "The idea of treatment is very simple: venous blood is taken from the baby, pumped round a circuit which includes a membrane where the blood is oxygenated, reheated to body temperature and passed back into one of the baby's carotid arteries — ${ }^{159}$ thus bypassing the baby's lungs, the immaturity of which is implicated in the persistent hypertension." 160

The mortality rate before ECMO of neonates born with persistent pulmonary hypertension was $80 \%$. ECMO produced a $80 \%$ survival rate. ${ }^{161}$ The treatment was immediately tremendously successful, as was predicted by the physicians who had designed it. Despite this huge immediate successes, it was deemed necessary to perform an RCT in order for the treatment to be implemented in other hospitals as well, and to convince the medical community that ECMO really was better than the current standard treatment. It is important to note here that ECMO was very successful but not without risks, one of these and the most severe being intercranial bleeding which can lead either to death or to a severe brain malfunction. The consequences of which can be a severe handicap, a markedly reduced life-span and a reduced quality of life. Since not treating these newborns would mean unavoidable death, the possibility of detrimental side-effects of the treatment versus the chance of a full recovery were certainly recognised but the severity of the possible sideeffects was deemed crucial enough to establish clinical equipoise with regard to ECMO. At least when following the argument of Robyn Bluhm. It is questionable in the case of ECMO if clinical equipoise was ever present. The inventors of ECMO believed in their new system and the survival rates proved them right. Other clinics could have been convinced by mechanistic reasoning through the method of ECMO and what it was supposed to treat. Robert Truog argued along those lines by claiming that it would have been enough to conduct a long-term observational study which would have shown ECMO to be superior to the standard treatment. ${ }^{162}$ Only a very stringent view as described above by Worrall, namely

159 http://commons.wikimedia.org/wiki/File:ECMO_schema-1-de.png License: This file is licensed under the Creative Commons Attribution-Share Alike 2.0 Germany license. Uploaded on November $14^{\text {th }}, 2019$.

160 John Worrall. (2008) Evidence and Ethics in Medicine. in Perspectives in Biology and Medicine. 51 (3): 419.

161 R. H. Bartlett, A. B. Gazzaniga. et.al. (1986). "Extracorporeal membrane oxygenation (ECMO) in neonatal respiratory failure. 100 cases." in Annals of Surgery. 204(3): 236-245.

162 Robert D Truog. (1999). "Informed consent and research design in critical care medicine." in Critical Care. 3(3):R29-R33. 
that there is no evidence without RCTs, did lead to the ECMO trials. And as has become clear, this stringent view is not necessarily the one that provides external validity of a treatment and the best possible outcome for the individual patient.

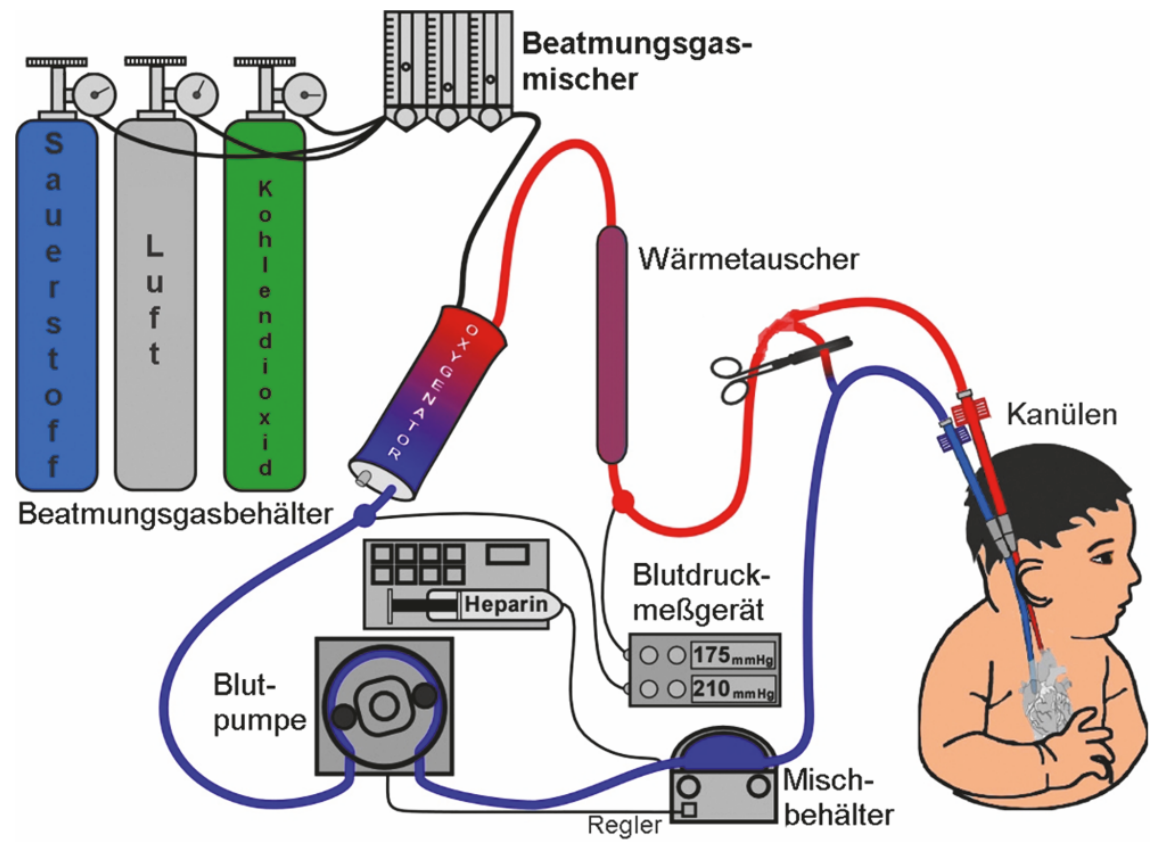

Figure 1: ECMO schema, to provide neonatal extracorporeal oxygenation

Source: https://upload.wikimedia.org/wikipedia/commons/thumb/f/fb/Ecmo_schema-1-de.png/1024pxEcmo schema-1-de.png. License: This file is licensed under the Creative Commons Attribution-Share Alike 2.0 Germany license. Last accessed on November $14^{\text {th }}, 2019$.

Three RCTs were performed in a fairly short amount of time, of which the first two were deemed to be flawed. The first RCT used a method of randomisation called 'randomised plays the winner,' a method of randomisation that happens on an ongoing basis while the trial is already in progress. ${ }^{163}$ Most often an urn or some such device is used, even if just computer generated, that contains an equal number of, for example red and white balls. Red in this example stands for ECMO, white for the standard treatment. The urn is re-stacked with balls depending on survival. If a child survives on ECMO, then a red ball is added, if it dies on the standard, again a red ball is added. This method produced a biased urn very quickly, because

163 Catherine Cornu et al. (2013). "Experimental designs for small randomised clinical trials: an algorithm for choice." in Orphanet Journal of Rare Diseases. 8(48): 4. 
the first child was assigned to ECMO and survived while the second was assigned to the standard and died. Since with such a heavily biased urn, a statistically sound assessment of the validity of the trial was not possible anymore, a second trial was deemed necessary.

The second trial used a different form of adaptive randomisation to begin with and then it used the Zelen method. For those reasons, and an incorrect use of stopping clauses, or so it was claimed, the trial was stopped early. ${ }^{164}$

A third trial was initiated. However, since some form of comparison was deemed necessary, the methods used in both arms of the trial were already outdated by the methods used to treat pulmonary hypertension in neonates in the every day clinical setting. The standard had become better and more successful over time, as had ECMO. ${ }^{165}$ Still, the outdated versions were used, solely for the sake of science and certainly not for the sake of the actual patients. It can be said without a doubt that any RCT conducted under these circumstances is not ethically correct and should not be permitted to be performed. Clinical equipoise cannot have been present anymore. The technique was already successfully used in many clinics and had therefore proven to be externally valid. The consent given by the participants of the last trial can not have been that informed, since there were better options available and to make matters even worse, the last trial did change nothing in the overall acceptance of ECMO in every day clinical practice. ${ }^{166}$

\subsection{Conclusion}

Medical research is important and can save lives. If done correctly, medical research is as safe as humanly possible and there are many solutions to translate the results of medical research into usable results for the individual patient. As we have seen with ECMO, medical research, and especially RCTs, should not be conducted just because the methodology is deemed superior. If other forms of evidence are available, like mechanistic reasoning, or the evidence from other forms of studies, and even experience, it should be taken into account and used wherever appropriate. This approach to evidence not only saves time and money but also patients from being subjected the superfluous trials or dangerous treatments. And patients and participants need their own voice in the medical process, not only in

164 Robyn Bluhm. (2010). "The epistemology and ethics of chronic disease research: Further lessons from ECMO.” in Theory of Medical Bioethics. 31:107-122.

165 Robert D Truog. (1999):R29-R33.

166 Valerie Mike, Alfred N Krauss, and Gail S Ross. (1993). "Neonatal extracorporeal membrane oxygenation (ECMO): clinical trials and the ethics of evidence." in Journal of medical ethics; 19: $212-218$ 
medical practice but also and especially in medical research, since without participants there would be no research. The tool of informed consent is what can provide this voice.

Open Access This chapter is licensed under the terms of the Creative Commons Attribution 4.0 International License (http://creativecommons.org/licenses/by/4.0/), which permits use, sharing, adaptation, distribution and reproduction in any medium or format, as long as you give appropriate credit to the original author(s) and the source, provide a link to the Creative Commons license and indicate if changes were made.

The images or other third party material in this chapter are included in the chapter's Creative Commons license, unless indicated otherwise in a credit line to the material. If material is not included in the chapter's Creative Commons license and your intended use is not permitted by statutory regulation or exceeds the permitted use, you will need to obtain permission directly from the copyright holder.. 